\title{
Epidemiology of acute spinal cord injuries in the Groote Schuur Hospital Acute Spinal Cord Injury (GSH ASCI) Unit, Cape Town, South Africa, over the past 11 years
}

\author{
J Sothmann, MB ChB; J Stander, MB ChB; N Kruger, BSc, MB ChB, FRCS (Ed), FCS Orth (SA); \\ R Dunn, MB ChB, MMed (Orth), FCOrth (SA) \\ Acute Spinal Injury Unit, Division of Orthopaedic Surgery, Groote Schuur Hospital and Faculty of Health Sciences, \\ University of Cape Town, South Africa
}

Corresponding author: J Sothmann (pjsoth@yahoo.co.uk)

\begin{abstract}
Background. Spinal cord injury (SCI) is devastating to both patient and society, with acute management and ongoing care being extremely expensive. Few epidemiological data are available on SCIs in South Africa (SA).

Objectives. To identify the epidemiological profile of SCI patients at Groote Schuur Hospital (GSH), Cape Town, SA, and identify seasonal trends and peak periods. As the majority of the injuries are preventable, these data are important to develop prevention strategies.

Methods. A retrospective review of prospectively collected data was conducted on all patients admitted to the Acute Spinal Cord Injury (ASCI) Unit at GSH from 1 April 2003 to 31 March 2014. All cases registered on a prospectively maintained database were included in the study.

Results. The total number of patients admitted to the ASCI Unit was 2 042, with an average of 185 admissions per year. The male/female ratio was 5.25:1. The 21 - 30-year-old age category was the largest, comprising $33.5 \%$ of the patients. The most prevalent cause of injury was motor vehicle accidents (44.6\%), followed by violence-related injuries (27.2\%). Thirty-two point two percent of patients needed ventilatory support, and $91.5 \%$ of mechanically ventilated patients were successfully weaned. December was the busiest month in the unit. In patients in whom neurological deficit was incomplete, the average motor function improvement was $16.0 \%$.

Conclusions. Data capturing and analysis of SCIs should be encouraged in SA to guide management and prevention strategies, and to optimise outcomes. This study establishes the ASCI Unit at GSH to be one of the key role players in acute SCI management in SA.
\end{abstract}

S Afr Med J 2015;105(10):835-839. DOI:10.7196/SAMJnew.8072

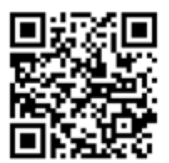

Spinal cord injury (SCI) is devastating to both the patient and society, with loss of productivity of this predominantly young and healthy age group and increased dependence on family and societal support. Acute management and ongoing care are extremely expensive ${ }^{[1]}$ Few epidemiological data are available on SCIs in South Africa (SA). Worldwide reviews ${ }^{[2-4]}$ often include SA data based on studies done two decades ago. ${ }^{[5-7]}$ Such data are important to develop preventive strategies, as many of these injuries are preventable.

\section{Objectives}

To identify the epidemiological profile of SCI patients at Groote Schuur Hospital (GSH), Cape Town, SA, identify seasonal trends and peak periods, and determine whether specific causes of injury are on the incline or decline.

\section{Methods}

The Acute Spinal Cord Injury (ASCI) Unit at GSH is a specialised unit for the management of patients from the Western Cape Province with acute traumatic spinal cord injuries. The unit has 21 beds, with 6 beds allocated to intensive care (4 ventilated and 2 non-ventilated). The mission of the unit is to manage the acute phase of the SCI, and its role is to stabilise patients from a mechanical and physiological point of view to optimise neurological outcome. The unit was established on 1 April 2003, and every admission since then has been documented on a prospective database.

We conducted a retrospective study on all patients admitted to the ASCI Unit at GSH from 1 April 2003 to 31 March 2014. Data were extracted from a prospectively maintained database in the unit, into which patient information is entered on discharge. All cases that were registered on the database were included in the study.

Information on age, gender, cause of injury, level of injury and referring hospitals was extracted from the database. Additional information that was extracted from the database was the proportion of patients who required surgery, whether ventilatory support was rendered, waiting periods for admission to the unit and duration of hospital stay. The waiting period before admission to the unit was calculated as the time between the date of the injury and the date of admission to the unit (expressed in weeks). The duration of stay in the unit was calculated as the time between the date of admission to the unit and the date of discharge (expressed in months).

Patients' neurological status was documented using the American Spinal Injury Association (ASIA) score. The total ASIA score (motor function plus sensation to soft touch plus sensation to pin prick) on admission was deducted from the total ASIA score on discharge from the unit, to determine the change in neurological status at the time of discharge.

Of the patients with improvement in total ASIA scores, those with motor function improvement were identified (motor function score out of 100). The average improvement in motor function on discharge was determined separately for patients with incomplete and complete neurological deficit (some preservation of neurological function below the level of SCI and no function below the level of the injury, respectively, with the level defined as the last normal functioning level, e.g. in C6 motor complete injury, wrist dorsiflexion is normal and below this level there is no motor function except for a possible zone of partial preservation corresponding with spinal cord 
oedema. In C6 motor incomplete injury, wrist dorsiflexion is normal and below this level there is preservation of some motor function down to the sacral segments).

Admission of patients to the unit was based on the ASCI Unit admission criteria and priority scale shown in Table 1.

\section{Ethics approval}

Ethics approval was obtained from the Human Research Ethics Committee of the Faculty of Health Sciences, University of Cape Town (HREC/REF: 405/2014).

\section{Results}

Total number of patients and gender

Between 1 April 2003 and 31 March 2014, 2042 patients were admitted to the ASCI Unit. This represents an average of 185 patients per year. Of the patients, $84.0 \%(n=1$ $715)$ were male and $16.0 \%(n=327)$ female. The male/female ratio was 5.25:1.

\section{Age}

The mean age of the patients was 34 years. The largest age category was 21 - 30 years (33.5\% $(n=684)$ of the total number of patients), followed by the 31 - 40-year age category $(25.2 \%, n=514)$ and the 41 - 50-year age category $(16.6 \%, n=339)$. Fig. 1 shows the breakdown of all patients by age group.

\section{Causes of SCIs}

The most prevalent cause of injury was motor vehicle accidents (MVAs), which accounted for $44.6 \%$ of all injuries $(n=910)$. Falls accounted for $15.5 \%$ of all injuries $(n=316)$ and gunshot wounds (GSWs) for $14.4 \%$ ( $n=294)$. Fig. 2 shows the causes of the injuries in descending order.

MVAs were divided into three categories (Fig. 3). MVA passenger accounted for $56.0 \%$ of the total number of MVA injuries $(n=510)$, MVA driver for $25.6 \%(n=233)$ and MVA pedestrian for $18.4 \%(n=167)$.

\section{Level of injury}

Of 2042 patients, $59.3 \%$ ( $n=1210)$ had cervical, $27.2 \%(n=556)$ thoracic and $11.2 \%(n=228)$ lumbosacral injuries. For the remaining 2.3\% $(n=48)$, no data were available.

\section{Referring hospitals}

The majority of the patients $(45.3 \%, n=925)$ were referred directly from $\mathrm{GSH}$, followed by $20.7 \% \quad(n=422)$ referred from Tygerberg Hospital and 10.7\% ( $n=218)$ from George Hospital. Most of the remaining referrals were from other hospitals in the Western Cape, with a small number of patients from other hospitals across SA. The top ten referring hospitals were GSH (45.3\%), Tygerberg Hospital (20.7\%),

Table 1. Acute spinal cord injury admissions to ASCI Unit, priority scale*

P1 Incomplete acute SCI in need of urgent spinal cord decompression operation/procedure

P2 Acute SCI with unstable cervical spine fracture in need of spinal fusion operation

P3 Acute SCI with unstable thoracic/lumbar spine fracture in need of spinal fusion operation

P4 Acute SCI with SCI-related complication e.g. spinal shock, respiratory failure

P5 Acute SCI with unstable fracture of the spine for conservative management in traction

P6 Acute SCI for removal of bullet

P7 Acute SCI with stable fracture of the spine

P8 Acute SCI, not fit for rehabilitation (e.g. infection or SCI-related complications)

P9 Stable post-acute SCI fit for rehabilitation

P10 Stable post-acute SCI with sacral, ischial or trochanteric pressure ulcer, otherwise fit for rehabilitation

*Inclusion criteria: acute traumatic spinal cord injury; exclusion criteria: severe head injury, Glasgow Coma Score $<13 / 15$ o $<9 \mathrm{~T} / 15$, polytrauma.

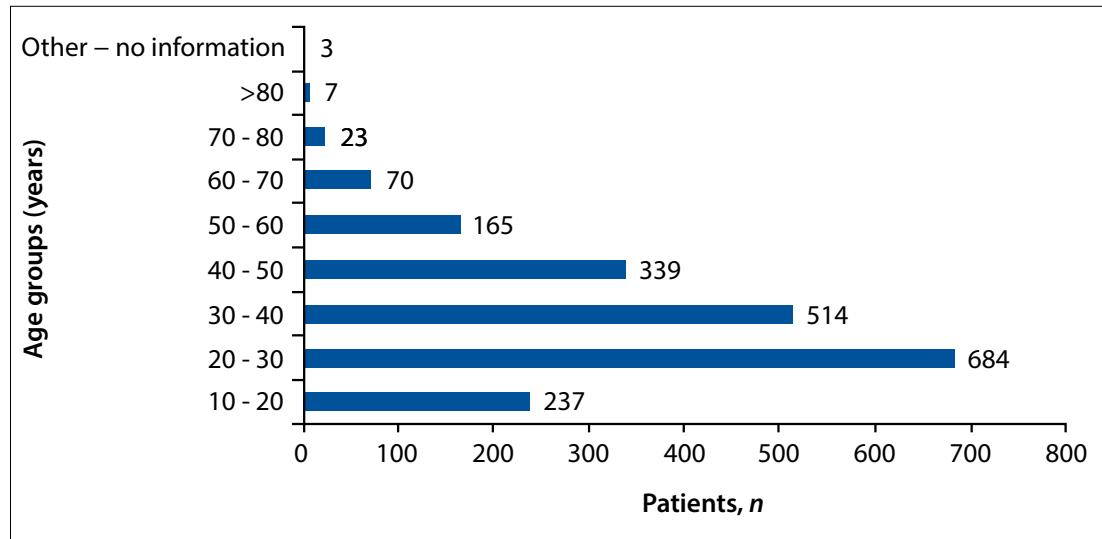

Fig. 1. All patients by age group.

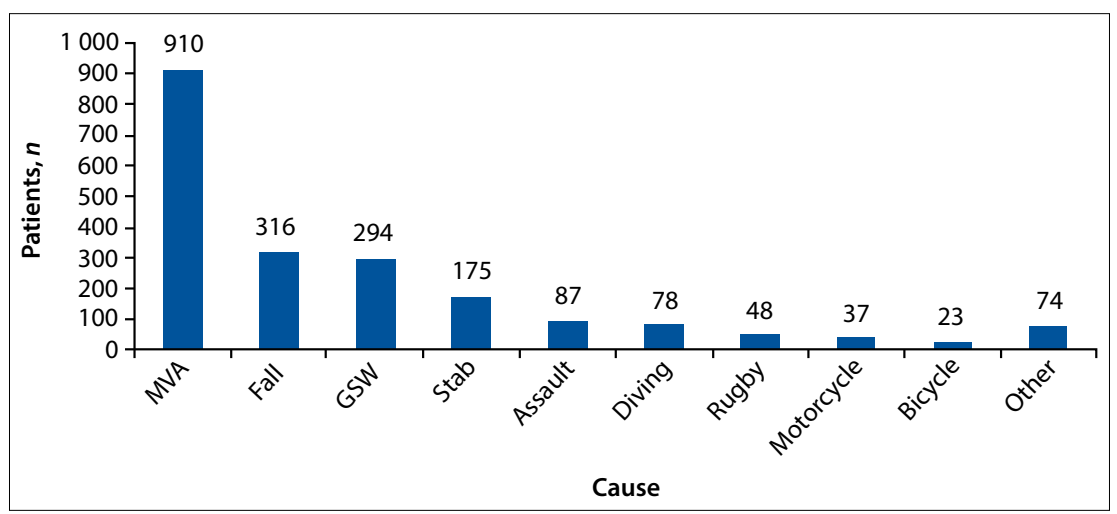

Fig. 2. All patients by cause.

George Hospital (10.7\%), New Somerset Hospital (2.7\%), G F Jooste Hospital (2.3\%), Worcester Hospital (2.1\%), Victoria Hospital (1.8\%), Vredenburg Hospital (1.5\%) and Paarl Hospital (1.1\%). The hospital was unknown in $1.0 \%$ of cases.

\section{Surgery and ventilation}

Of the patients, $64.6 \%$ underwent spinal surgery, $23.4 \%$ required mechanical ventilation, and $8.8 \%$ required non-invasive ventilatory support (32.2\% in total requiring ventilatory support).
Of the mechanically ventilated patients, $91.5 \%$ were successfully weaned off ventilation, $0.9 \%$ remained ventilator dependent, and $7.6 \%$ died.

\section{Mortality rate}

The mortality rate of the ASCI Unit inpatients was $2.2 \%$.

\section{Waiting periods and hospital stay}

Of the patients, $69.9 \% \quad(n=1427)$ were admitted within the first week of injury, 
$13.4 \%(n=274)$ within the second week, $5.5 \%$ $(n=113)$ within the third week and $3.2 \%$ ( $n=66)$ within the fourth week. In 154 cases $(7.5 \%)$ there was a delay of more than one month before admission (Fig. 4).

Regarding length of stay in the unit, $64.2 \%$ $(n=1310)$ of the total number of patients stayed for $<1$ month, $21.9 \% \quad(n=448)$ of patients for $1-2$ months, $6.1 \% \quad(n=124)$ for 2 - 3 months and $7.5 \%(n=154)$ for $>3$ months (Fig. 5). The average length of stay in the unit was 28 days.

\section{Neurological status}

On admission, $31.7 \%$ of patients had complete and $68.3 \%$ incomplete spinal cord injuries.

In $50.7 \% \quad(n=1 \quad 035)$ there was an improvement in total ASIA score by the time of discharge from the unit, in $39.1 \%(n=798)$ the ASIA score remained the same, and in $10.2 \%(n=209)$ there was a deterioration in ASIA score by the time of discharge.

Of the patients who experienced an improvement in total ASIA score, $85.4 \%$ $(n=884)$ showed improvement in motor function. Motor function in patients with incomplete neurological deficit improved by an average of 16 (median improvement 10, interquartile range 5 - 22). Motor function in patients with complete neurological deficit improved by an average of 8 (median improvement 5 , interquartile range $3-8$ ).

\section{Trend analysis}

The total number of admissions was evaluated according to the month of admission in an effort to determine a monthly admission trend. Fig. 6 shows all patient admissions per month over 11 years.

Analysis of causes of injury over the years revealed the trends shown in Fig. 7, a and b.

\section{Discussion}

Our patient profile mirrors international literature in terms of gender and age, with a male/female ratio of $5.25: 1^{[3]}$ and age distribution and mean age being similar to other studies, with the 21 - 30-year category the largest $(33.5 \%))^{[3,5]}$

Similarly, the causes of injuries in this study compare with the international literature, with MVAs and falls being the two largest categories. $^{[2-4,8]}$ Previous SA literature described violence as the number one cause of $\mathrm{SCI} \cdot{ }^{[5,7]}$ This could be influenced by grouping causes. If we add our study results for violence-related causes (GSWs plus stabs plus blunt assault), violence accounts for $27.2 \%$ of injuries. Violence-related SCI therefore remains second to MVAs as a cause of SCI in the ASCI Unit. Prevention strategies are currently focused on drivers, yet pedestrians accounted for $18.4 \%$ of the total number of MVAs (and $8.2 \%$ of patients in total), which is related to alcohol abuse and disregard for road safety. It is clear that there needs to be a focus in this area as an important component of MVA prevention campaigns. Since falls were a significantly large aetiological category of SCI (third after MVAs and violence), further investigation regarding the circumstances of falls is needed to determine where prevention should be aimed, e.g. at work safety regulations, falls in the elderly, etc.

The $3.6 \%(n=74)$ of remaining injuries included a spectrum of causes such as train accidents, wrestling injuries, objects falling onto patients, aeroplane accidents, paragliding accidents, horse riding accidents, surfing and angle grinder accidents. Other

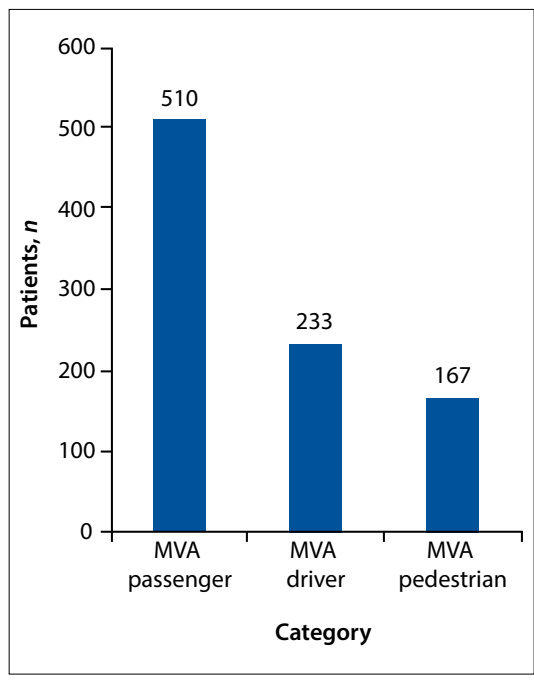

Fig. 3. MVA categories.

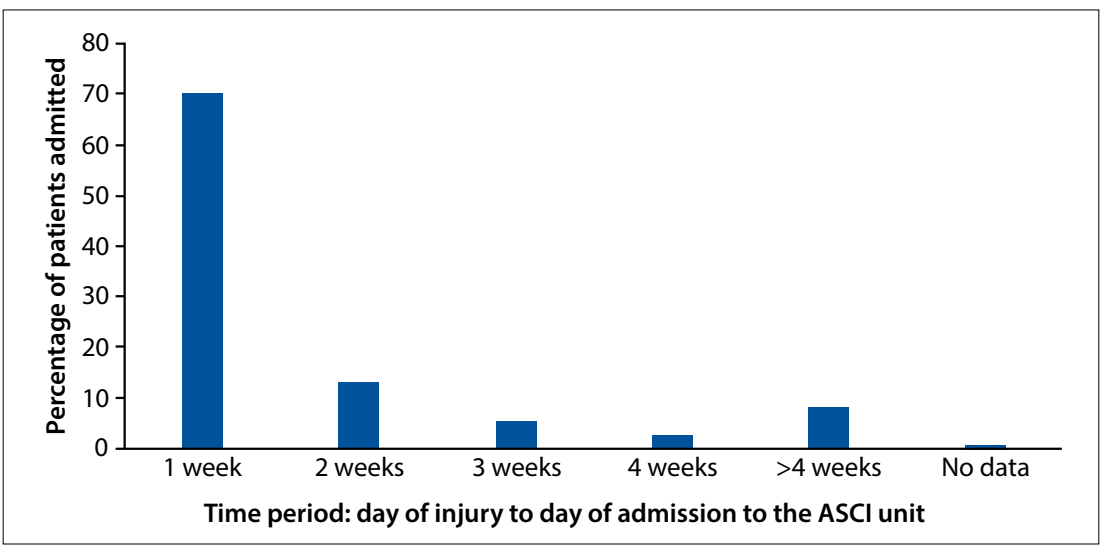

Fig. 4. Time period to admission after injury.

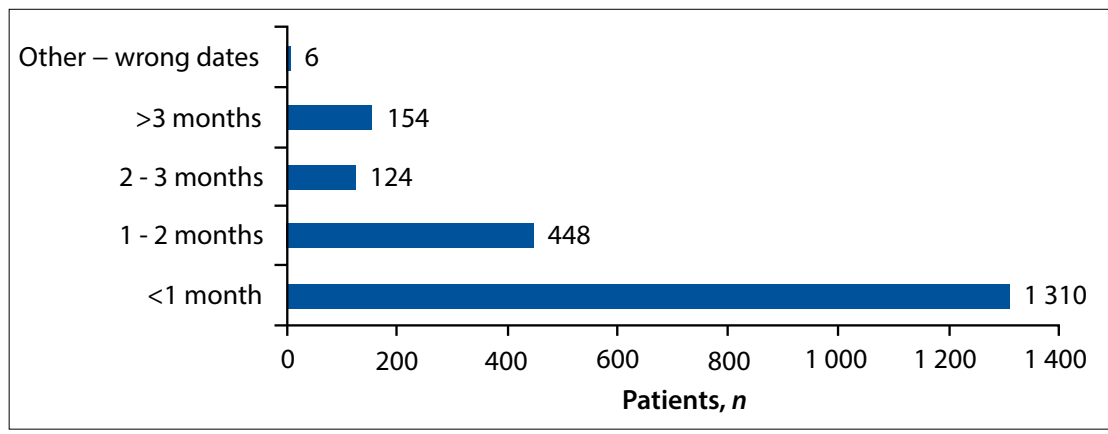

Fig. 5. Length of stay.

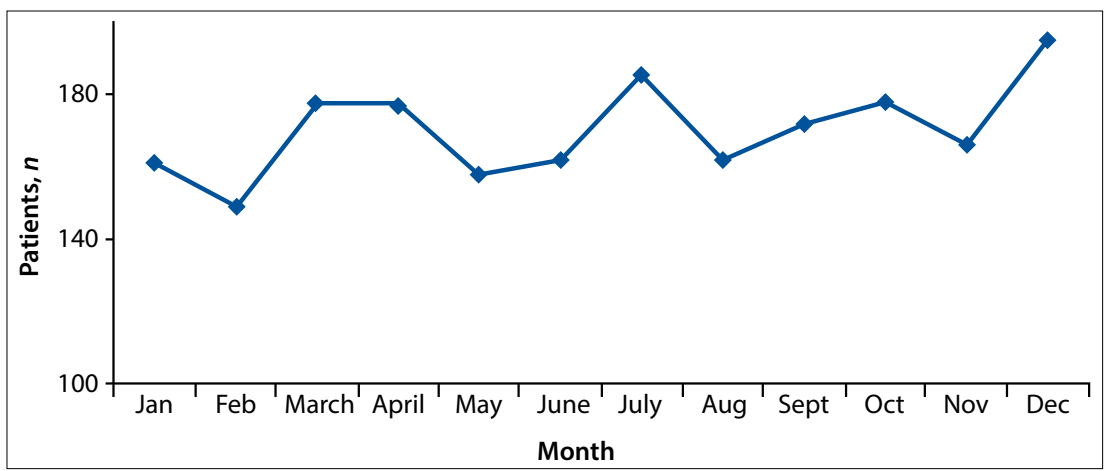

Fig. 6. All patient admissions per month (age $>11$ years). 


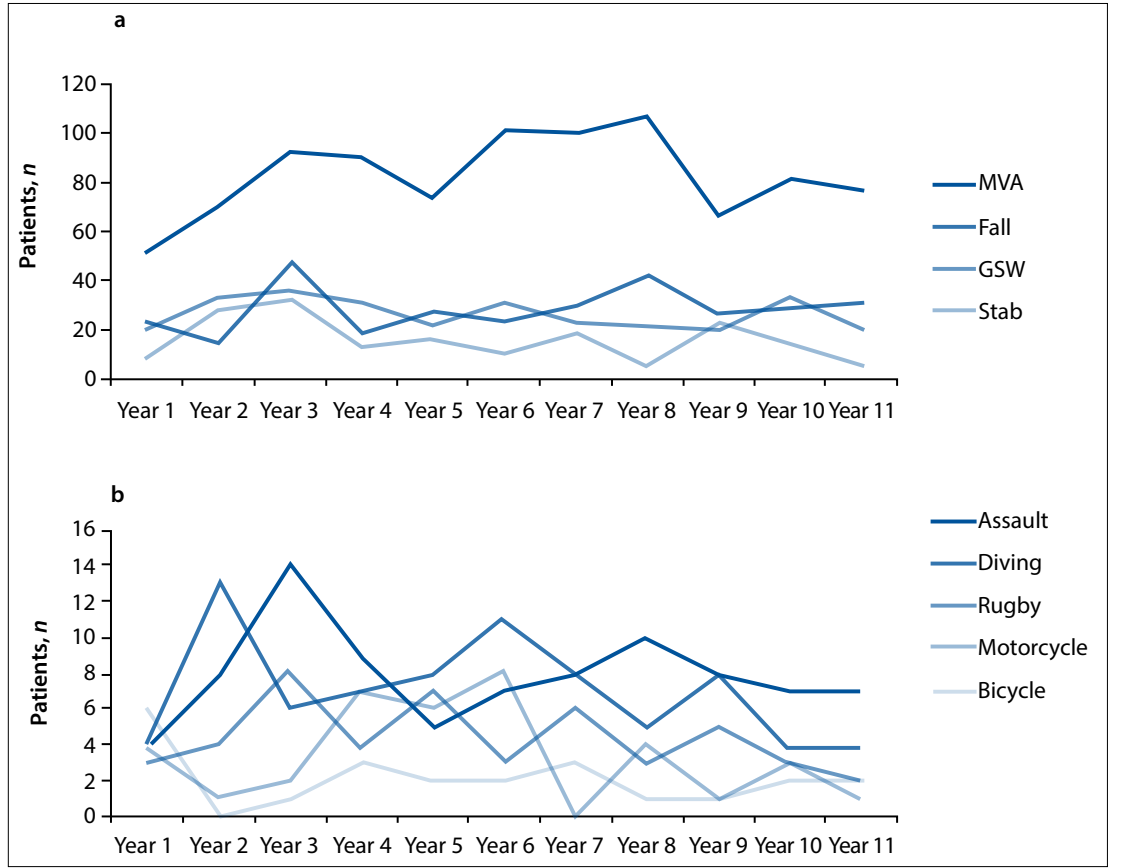

Fig. 7. Yearly trends for causes: (a) MVAs, falls, GSWs and stab wounds; (b) assaults and diving, rugby, motorcycle and bicycle accidents.

causes for admission included readmissions for special investigations, and in some cases no specific information was available on the cause of injury. An additional 84 patients were managed in the unit but could not be included in the study owing to incomplete data capturing.

Admissions are prioritised according to the severity of the SCI, the need for decompression/surgery and potential neurological outcome. Owing to our unit's prioritisation of admission of patients requiring supportive care, the profile may not fully represent the SCI profile. As more SCI patients are referred to the unit than can be accommodated, stable patients and those who are not in need of surgery are redirected to the local rehabilitation centre. This could account for the highest percentage of injuries being cervical SCIs (59.3\%), as these injuries are associated with a higher incidence of complications than thoracic and lumbosacral injuries. This would also account for the high incidence of patients in need of ventilatory support, $32.2 \%$ in total (ventilated patients as well as non-invasive ventilator support) For $2.3 \%$ of the patients no specific data were available on the level of injury.

Of the total number of ventilated patients, $0.9 \%$ were ventilator dependent at discharge and transferred to a rehabilitation facility on a mobile ventilator.

Our study shows that the majority of patients (69.9\%) were admitted to the unit within 1 week. The total proportion of patients admitted within 2 weeks was
83.3\%. Admissions are prioritised according to previously mentioned criteria, which explains the longer waiting periods for uncomplicated or stable injuries.

Length of patients' stay in hospital affects the ability of the unit to admit new patients. The length of stay for $64.2 \%$ of patients was $<1$ month, and $7.5 \%$ stayed in hospital for $>3$ months. Patients requiring a prolonged stay (>3 months) mainly comprised those with complications such as pressure ulcers that limited their progression to rehabilitation (this is worthy of future research).

SCI mortality rates differ between countries and regions, with available data suggesting that mortality, calculated as death in the first year after injury, ranges from $4.0 \%$ (North America) to $84.0 \%$ (West Africa). ${ }^{[9]}$ The low mortality rate of $2.2 \%$ in the GSH ASCI Unit reflects the rate calculated at discharge from the post-acute ward.

Neurological recovery after a traumatic SCI is influenced by the cause and severity of the injury. ${ }^{[10]}$ The motor function ASIA score was extracted because it correlates with functionality. The motor function improvement data followed an abnormal distribution pattern, but it needs to be remembered that the reported improvement reflects only the acute phase, over an average admission period of 28 days (admission to discharge from post-acute ward), while neurological recovery can be expected for 5 years and longer after injury. ${ }^{[11,12]}$

December is the busiest month of the year in the unit. Other peak periods (Fig. 6) coincided with school holidays - the Western Cape is a popular holiday destination during holiday seasons, with additional road traffic load. The ASCI Unit admission criteria and priority scale proved especially useful during these periods, when new referrals tend to exceed available resources.

The top three causes of SCIs (MVAs, falls, GSWs) showed a slight incline per year, following the opening of our unit (Fig. 7a). That stab wounds showed a decline could be explained by the fact that the majority of patients with isolated SCI secondary to a stab wound tend to be fairly stable and are referred directly to a rehabilitation centre, bypassing the ASCI Unit. Fig. 7b shows that assaults remained stable, diving injuries showed a decline (possibly as result of active prevention strategies), rugby injuries showed a steady decline (reflecting good practices and prevention campaigns such as Boksmart by SA Rugby), motorcycle accidents decreased, and bicycle accidents stayed the same.

\section{Conclusion}

There are limited data available on the causes of SCIs in SA. The finding of MVAs as the top cause of SCIs seen in the ASCI Unit at GSH indicates the thrust of prevention strategies. More emphasis should be on all road users, including pedestrians. Prevention campaigns directed at MVAs should be re-evaluated and learn from other campaigns, such as rugby and diving, which have resulted in a significant decline in the incidence of injury.

Violence, identified as the second most prevalent cause of traumatic SCIs, calls for a focused, effective prevention campaign.

The evidence that there are obvious peak periods of SCI, as identified over the past 11 years, suggests motivation for additional beds to be made available to the unit during these periods.

These data create a platform for further studies that could be helpful in many aspects of preventing SCIs. This information could be used by the Western Cape Provincial Government to determine the incidence of SCI for the province and where prevention campaigns should be aimed, while emphasising the importance for adequate facilities for the disabled. One aspect that calls for further study is comparison of the cost of SCIs with the cost of prevention campaigns.

Strategies should be implemented to encourage SCI, trauma and rehabilitation centres to create methods of data collection, as very few data are available regarding the causes of SCIs in other provinces of SA. 
Our experience of SCIs, showing a low mortality rate of $2.2 \%$, a successful weaning rate of $91.5 \%$ and average motor function improvement in incomplete injuries of $16.0 \%$, reveals the clear advantages of a dedicated spinal unit.

\section{References}

1. Mahabaleshwarkar R, Khanna R. National hospitalization burden associated with spinal cord injuries in the United States. Spinal Cord 2014;52(2):139-144. [http://dx.doi.org/10.1038/sc.2013.144]

2. Cripps RA, Lee BB, Wing P, Weerts E, Mackay J, Brown D. A global map for traumatic spinal cord injury epidemiology: Towards a living data repository for injury prevention. Spinal Cord 2011;49(4):493-501. http://dx.doi.org/10.1038/sc.2010.146]

3. Ackery A, Tator C, Krassioukov A. A global perspective on spinal cord injury epidemiology. J Neurotrauma 2004;21(10):1355-1370. [http://dx.doi.org/10.1089/neu.2004.21.1355]

4. Draulans N, Kiekens C, Roels E, Peers K. Etiology of spinal cord injuries in sub-Saharan Africa. Spinal Cord 2011;49(12):1148-1154. [http://dx.doi.org/10.1038/sc.2011.93]

5. Hart C, Williams E. Epidemiology of spinal cord injuries: A reflection of change in South African society. Paraplegia 1994;32(11):709-714. [http://dx.doi.org/10.1038/sc.1994.115]
6. Odendaal T. Injuries of the cervical spinal cord. S Afr Med J 1991;80(2):75-79.

7. Velmahos GC, Degiannis E, Hart K, Souter I, Saadia R. Changing profiles in spinal cord injuries and risk factors influencing recovery after penetrating injuries. I Trauma 1995;38(3):334-337. [http:// dx.doi.org/10.1097/00005373-199503000-0004]

8. International Spinal Cord Society. SCI global mapping. Archive global data TSCI. Supplementary Table $2-20 / 8 / 2010$. Incidence and aetiology of traumatic SCI by region and author(s) of published data. http://iscos.org.uk/page.php?content=57 (accessed 2 February 2015).

9. International Spinal Cord Society. SCI global mapping. Archive Global data TSCI. Supplementary

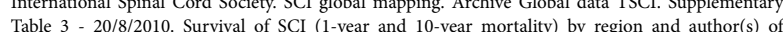
published data. http://iscos.org.uk/page.php?content=57 (accessed 2 February 2015).

10. Marino RJ, Ditunno JF Jr, Donovan WH, Maynard F Jr. Neurologic recovery after traumatic spinal cord Marino RJ, Ditunno JF Jr, Donovan WH, Maynard FJr. Neurologic recovery after traumatic spinal cord
injury: Data from the Model Spinal Cord Injury Systems. Arch Phys Med Rehabil 1999;80(11):1391injury: Data from the Model Spinal Cord Injury Systems. Arch Phys Med Rehabil 1999;80(11):13911396. [http://dx.doi.org/10.1016/S003-9993(99)90249-6]

1. Kirshblum S, Millis S, McKinley W, Tulsky D. Late neurologic recovery after traumatic spinal cor injury. Arch Phys Med Rehabil 2004;85(11):1811-1817. [http://dx.doi.org/10.1016/j.apmr.2004.03.015]

12. Choe AS, Belegu V, Yoshida S, et al. Extensive neurological recovery from a complete spinal cord injury: A case report and hypothesis on the role of cortical plasticity. Front Hum Neurosci 2013;7:290. [http://dx.doi.org/10.3389/fnhum.2013.00290]

Accepted 29 June 2015 